\title{
Fugue et variation sur un même thème
}

\author{
Nadine Ltaif
}

\section{Fugue and Variation on the Same Theme}

In the Ethics of Sexual Difference, drawing on the thought of Emmanuel Levinas, Luce Irigary explores the notion of an ethical imperative to confront otherness in all of its inconceivability, rather than making the other a mirror for the projections of the self. In other words, there is an ethical imperative to confront the otherness within the self rather than projecting it onto another. The paradigmatic figure of this ethical relation, the face-à-face, presents itself early in Ltaif's text; the woman in the speaker's recurrent dream lives in the house across from that of the speaker. In the dream-fugue, the woman alternately is blind to the speaker and smiles beckoningly; one night, the woman steps into the space between them and comes toward the speaker, a frightening gesture that nevertheless eases the speaker's sense of being at a loss in waking hours. In the variation of the dream, the speaker can no longer feel the presence of the other woman and remembers with a sense of urgency a time when she had saved her from an overdose. This time the oscillation is between the desire to call the woman, to help her, and a strong sense that she must leave her alone. Addressing the other woman, telling her she can no longer help her, not only brings the speaker a sense of peace but also brings her face to face with the other woman, who smiles and gives her a knowing wink.

\section{Fugue}

Il y a ce rêve que je fais périodiquement. Ce rêve qui me revient. Je me retrouve sur notre terrasse à Beyrouth ouest. Elle, elle habite en face, dans la maison d'une amie d'enfance. C'est sa maison. Nos balcons se font face. Comme des miroirs. Elle apparaît, étendue dans son lit. Étrangement, la seule chose qu'il y a dans cette chambre est un lit. Nous avions comme fantasme, mon amie et moi, de communiquer par une corde que nous étendions entre les deux immeubles. Nous n'avions jamais réalisé ce rêve mais nous utilisions nos voix et nos noms que nous 
psalmodions comme les muezzins du vendredi. Rien. Elle ne semblait pas me voir. Puis, c'est elle qui semble consentante. Elle me sourit. Toujours le même rêve l'espace de quelques secondes. Et puis, une nuit, elle fait le saut dans le vide, s'avance vers moi. Je suis terrorisée de vivre une telle jouissance. Il y a des jours où je me sens tellement en manque que les rares moments de bonheur restés dans ma tête sont notre unique refuge. Heureusement, il y a sur nos déserts des mirages. Je la remercie mille fois de m'avoir donné de la rêver. Puisqu'il n'y a plus que le rêve où nous esquivons la loi.

\section{Variation}

J'ai eu encore une vision. Vers 21 heures je devais m'isoler. C'était un jeudi je crois ou un vendredi soir. Et là, je ne sentais plus sa présence et j'étais affolée. J'essayais de la rejoindre en pensée et je ne le pouvais plus. Il y avait un cordon coupé. Alors, $j$ 'ai tellement eu peur, je croyais qu'elle était en train de sombrer dans un sommeil sans fin. Je croyais qu'elle voulait que je l'appelle, que je devais absolument le faire, pour la sauver. En même temps. Une force nouvelle $\mathrm{m}^{\prime}$ interdisait de le faire. Il fallait que je la laisse, que je l'abandonne. C'était réel, car tout l'est. Je lui parlais, «je ne peux rien pour toi, je ne peux pas t'aider». Je souffrais de tiraillement impossible. Je me souviens du jour où je l'avais sauvée d'un sommeil bizarre. Elle avait pris trop de pilules. «Ma chère dame, je ne peux plus rien pour vous». Et voilà que je ne souffrais déjà plus. L'apaisement avait remplacé le tourbillon. Cela avait duré dix minutes. Je continuais de lui parler: «Va, je suis heureuse, je n'ai plus besoin de toi. Va tu es heureuse aussi». Et alors elle m'apparut. Le danger était passé. Je l'avais sauvée encore une fois, sans même l'appeler. Devenue sorcière. Elle m'apparaissait et me clignait des yeux d'un air complice comme elle le faisait en esquissant un bref sourire. Un sourire heureux. Et alors, la joie m'inondait et le rire. Je vivais mon deuil, mon œedipe. Oui, elle n'avait ni besoin de moi. Ni moi d'elle. 